
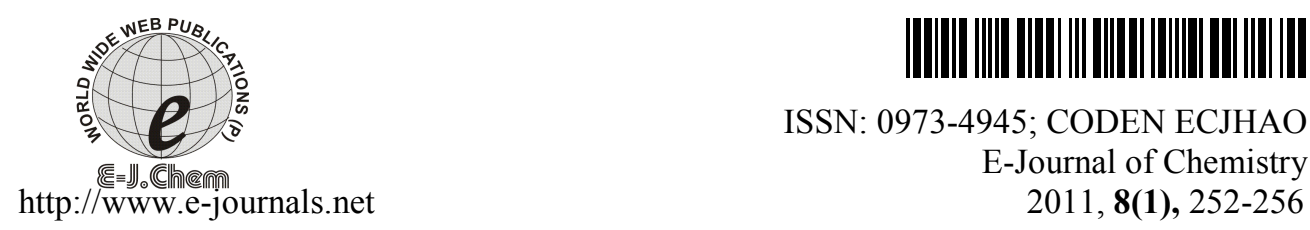

ISSN: 0973-4945; CODEN ECJHAO

E-Journal of Chemistry $2011, \mathbf{8}(\mathbf{1}), 252-256$

\title{
Theoretical Evaluation of Ultrasonic Velocities in Ternary Liquid Mixtures of Dibutylether + Toluene + 1-Alkanols at 303K
}

\author{
T. SUMATHI* and K. ABEETHA \\ Department of Physics (DDE) \\ Annamalai University, Annamalai Nagar- 608 002, India \\ tsumathi92@yahoo.com
}

Received 1 May 2010; Accepted 18 July 2010

\begin{abstract}
A comparison of ultrasonic velocity evaluated from Nomoto's relation, Van Deal- Vangeel ideal mixtures relation, impedance dependence relation and collision factor theories with experimental values have been made in three ternary systems of 1-alkanol with common binary mixture of toluene and dibutylether at $303 \mathrm{~K}$. The relative applicability of these theories to the present systems has been checked and discussed.
\end{abstract}

Keywords: Ternary mixture, Ultrasonic velocity, Molecular interaction, Theoretical evaluation.

\section{Introduction}

The ultrasonic velocity measurement plays an important role in understanding the molecular interaction between the components of the mixture and provides an insight into the physicochemical properties of liquid mixtures such as molecular association and dissociation as well as the strength of interaction between the components ${ }^{1-2}$. In order to get more information, an attempt has been made to correlate the experimental findings with those predicted theoretically on the basis of molecular models ${ }^{3-6}$. Further, the best suitable theory for the given molecular system understudy is also done by calculating the average percentage error. The present work includes

$\begin{array}{ll}\text { System I } & \text { 1-Propanol + Toluene + Dibutylether (DBE) } \\ \text { System II } & \text { 1-Butanol + Toluene + Dibutylether (DBE) } \\ \text { System III } & \text { 1-Pentanol + Toluene + Dibutylether (DBE) }\end{array}$

\section{Experimental}

The chemicals used in the present work were analytical reagent (AR) and spectroscopic reagent (SR) grades with minimum assay of $99.9 \%$. In all systems, the various concentrations 
of the ternary liquid mixtures were prepared interms of mole fraction, out of which, the mole fraction of the second component toluene $\left(\mathrm{X}_{2}=0.4\right)$ was kept fixed while the mole fractions of the remaining two components 1-alkanols $\left(\mathrm{X}_{1}\right)$ and $\operatorname{DBE}\left(\mathrm{X}_{3}\right)$ were varied from 0.0 to 0.6. The density of pure liquids and liquid mixtures were determined using a pycknometer and gave an estimated reproducibility of $\pm 0.0001 \mathrm{gcm}^{-3}$. The ultrasonic velocity was measured by using an ultrasonic interferometer (Model: F81) supplied by M/S Mittal Enterprises, New Delhi, having the frequency $3 \mathrm{MHz}$ with an overall accuracy of $\pm 0.1 \mathrm{~ms}^{-1}$.

Theory

Experimentally measured ultrasonic velocity has been compared with those obtained from the following equations ${ }^{7-10}$;

Nomoto's relation (NR)

$$
U_{N R}=\left(\frac{X_{1} R_{1}+X_{2} R_{2}+X_{3} R_{3}}{X_{1} V_{1}+X_{2} V_{2}+X_{3} V_{3}}\right)^{3}
$$

Where $X_{1}, X_{2}$ and $X_{3}$ are the mole fractions of the components, $\mathrm{R}_{1}, \mathrm{R}_{2}$ and $\mathrm{R}_{3}$ respective molar sound velocities and $V_{1}, V_{2}$ and $V_{3}$ are the molar volumes respectively.

Where molar sound velocity $R=\frac{M}{\rho} U^{1 / 3}=V U^{1 / 3}$

The molar volume $V=\frac{M}{\rho}$

Ideal mixture relation (IMR)

$$
U_{I M R}=\left(\frac{1}{X_{1} m_{1}+X_{2} m_{2}+X_{3} m_{3}}\right)^{1 / 2}\left(\frac{X_{1}}{m_{1} U_{1}^{2}}+\frac{X_{2}}{m_{2} U_{2}{ }^{2}}+\frac{X_{3}}{m_{3} U_{3}^{2}}\right)^{-1 / 2}
$$

The degree of molecular interaction $\alpha$ can be computed from the equation given under,

$$
\alpha=\left(\frac{U^{2} \exp }{U^{2}{ }_{I M R}}\right)-1
$$

Impedance dependence relation (IDR)

$$
U_{I D R}=\frac{\sum_{I=1}^{3} X_{i} Z i}{\sum_{i=1}^{3} X i \bar{n} i}
$$

Where $Z$ is the acoustic impedance and $\rho$ is the density of the components in the mixture.

Collision factor theory (CFT)

$$
U_{C F T}=U_{\infty}\left[\frac{\left(X_{1} S_{1}+X_{2} S_{2}+X_{3} S_{3}\right)\left(X_{1} B_{1}+X_{2} B_{2}+X_{3} B_{3}\right)}{V m}\right]
$$

Where, Molar volume of mixture $V_{m}=\left[\frac{\left(X_{1} m_{1}+X_{2} m_{2}+X_{3} m_{3}\right)}{\rho}\right]$ Actual Volume $B=4 / 3 \pi r^{3} N$ 


$$
\text { Molecular radius } \quad r=\left(\frac{3 b}{16 \pi \mathrm{N}}\right)^{1 / 3}
$$

Where $U_{\infty}$ is the temperature dependent constant $\left(U_{\infty}=1600 \mathrm{~m} \mathrm{~s}^{-1}\right), \mathrm{b}$ is the Vander Waal's constant and $\mathrm{N}$ is the Avagadro Number

\section{Percentage deviation}

The percentage deviation in sound velocity between the experimental and computed values are calculated as

$$
\left(\frac{\Delta U}{U}\right) \%=\left(\frac{U_{\text {exp }}-U_{\text {theo }}}{U_{\text {exp }}}\right) 100
$$

Standard percentage error

The standard percentage error (e) is calculated using the relation

$$
\mathrm{e}=\left(\frac{\left(\sum \frac{\Delta \mathrm{U}}{\mathrm{U}}\right)^{2} \%}{\mathrm{n}}\right)^{1 / 2}
$$

\section{Results and Discussion}

The experimentally measured velocity and the estimated ultrasonic velocity from the various theoretical models for three systems are summarized in the Table 1. The limitations and approximations incorporated in these theories are responsible for it.

Table 1. Values of experimental, theoretical ultrasonic velocity (U) at $303 \mathrm{~K}$

\begin{tabular}{ccccccc}
\hline \multicolumn{6}{c}{ Mole fraction } & \multicolumn{5}{c}{ Ultrasonic velocity, $\mathrm{ms}^{-1}$} \\
\hline $\mathrm{X}_{1}$ & $\mathrm{X}_{3}$ & $\mathrm{U}_{\text {exp }}$ & $\mathrm{U}_{\mathrm{NR}}$ & $\mathrm{U}_{\text {IMR }}$ & $\mathrm{U}_{\mathrm{IDR}}$ & $\mathrm{U}_{\mathrm{CFT}}$ \\
\hline \multicolumn{6}{c}{ System I-Propanol +Toluene + Dibutylether } \\
\hline 0.0000 & 0.6000 & 1181.9 & 1180.9 & 1185.4 & 1200.4 & 1195.8 \\
0.0999 & 0.5000 & 1185.9 & 1186.3 & 1169.4 & 1204.9 & 1195.4 \\
0.2000 & 0.4000 & 1190.0 & 1192.5 & 1160.1 & 1209.3 & 1201.3 \\
0.3000 & 0.3000 & 1197.7 & 1199.7 & 1157.6 & 1213.8 & 1206.0 \\
0.4000 & 0.2000 & 1204.4 & 1208.4 & 1161.4 & 1218.1 & 1210.1 \\
0.5000 & 0.1000 & 1217.2 & 1218.7 & 1171.7 & 1222.5 & 1214.8 \\
0.6000 & 0.0000 & 1225.7 & 1231.4 & 1189.3 & 1226.9 & 1216.1 \\
0.0000 & 0.6000 & 1181.9 & 1180.9 & 1185.4 & 1200.4 & 1195.8 \\
0.1000 & 0.5000 & 1186.4 & 1188.4 & 1185.7 & 1208.1 & 1202.2 \\
0.2000 & 0.4000 & 1196.4 & 1197.0 & 1189.0 & 1215.7 & 1211.7 \\
0.3000 & 0.3000 & 1200.7 & 1206.6 & 1195.3 & 1223.3 & 1217.4 \\
0.4000 & 0.2000 & 1211.8 & 1217.7 & 1204.8 & 1230.7 & 1226.7 \\
0.5000 & 0.1000 & 1222.9 & 1230.5 & 1217.6 & 1238.1 & 1234.5 \\
0.6000 & 0.0000 & 1236.6 & 1245.5 & 1234.1 & 1245.4 & 1240.0 \\
0.0000 & 0.6000 & 1181.9 & 1180.9 & 1185.4 & 1200.4 & 1195.8 \\
0.1001 & 0.5000 & 1191.2 & 1191.3 & 1194.8 & 1211.3 & 1207.3 \\
0.2000 & 0.4001 & 1202.6 & 1202.8 & 1205.7 & 1222.1 & 1221.2 \\
0.3000 & 0.3000 & 1216.7 & 1215.4 & 1217.9 & 1232.8 & 1232.0 \\
0.4000 & 0.2000 & 1228.1 & 1229.5 & 1231.4 & 1243.4 & 1242.0 \\
0.5000 & 0.1000 & 1247.0 & 1231.6 & 1121.1 & 1244.5 & 1379.1 \\
0.6000 & 0.0000 & 1255.3 & 1262.9 & 1263.3 & 1264.2 & 1261.2 \\
\hline \multicolumn{7}{c}{ System II-Butanol +Toluene + Dibutylether }
\end{tabular}


It is assumed that all the molecules are spherical in shape, which is not true every time. According to Nomoto's theory, it is assumed that the volume does not change on mixing. Therefore, no interaction between the components of liquid mixtures has been taken into account. Similarly, the assumption for the formation of ideal mixing relation is that, the ratios of specific heats of ideal mixtures and the volumes are also equal. Again, no molecular interaction is taken into account. But on mixing two liquids, the interaction takes place because of various forces such as dispersion forces, charge transfer, hydrogen bonding, dipole-dipole and dipole-induced dipole interactions. Thus the observed deviation of theoretical values of velocity from the experimental values shows that the molecular interaction is taking place between the unlike molecules in the liquid mixture ${ }^{4}$.

Table 2. Values of the Modulus of percentage deviation, Average Percentage error and standard percentage error at $303 \mathrm{~K}$

\begin{tabular}{|c|c|c|c|c|}
\hline & $\mathrm{U}_{\mathrm{NR}}$ & $\mathrm{U}_{\mathrm{IMR}}$ & $\mathrm{U}_{\mathrm{IDR}}$ & $\mathrm{U}_{\mathrm{CFT}}$ \\
\hline \multicolumn{5}{|c|}{ System I-Propanol +Toluene + Dibutylether } \\
\hline & 0.09 & 0.29 & 1.56 & 1.18 \\
\hline & 0.12 & 1.31 & 1.69 & 0.89 \\
\hline & 0.21 & 2.51 & 1.63 & 0.96 \\
\hline & 0.17 & 3.34 & 1.34 & 0.69 \\
\hline & 0.33 & 3.57 & 1.15 & 0.48 \\
\hline & 0.13 & 3.73 & 0.44 & 0.20 \\
\hline & 0.47 & 2.96 & 0.09 & 0.78 \\
\hline APE & 0.22 & 2.53 & 1.13 & 0.74 \\
\hline SPE & 0.25 & 2.79 & 1.27 & 0.80 \\
\hline \multicolumn{5}{|c|}{ System II-Butanol +Toluene + Dibutylether } \\
\hline & 0.08 & 0.28 & 1.56 & 1.19 \\
\hline & 0.17 & 0.06 & 1.83 & 1.33 \\
\hline & 0.05 & 0.62 & 1.61 & 1.28 \\
\hline & 0.50 & 0.45 & 1.88 & 1.39 \\
\hline & 0.49 & 0.58 & 1.56 & 1.23 \\
\hline & 0.62 & 0.43 & 1.24 & 0.95 \\
\hline & 0.72 & 0.20 & 0.71 & 0.28 \\
\hline APE & 0.38 & 0.37 & 1.48 & 0.89 \\
\hline SPE & 0.45 & 0.42 & 1.53 & 1.15 \\
\hline \multicolumn{5}{|c|}{ System III-Pentanol+Toluene + Dibutylether } \\
\hline & 0.08 & 0.28 & 1.56 & 1.19 \\
\hline & 0.01 & 0.31 & 1.69 & 1.35 \\
\hline & 0.01 & 0.25 & 1.62 & 1.54 \\
\hline & 0.11 & 0.10 & 1.33 & 1.26 \\
\hline & 0.11 & 0.27 & 1.25 & 1.13 \\
\hline & 1.24 & 10.1 & 0.20 & 10.6 \\
\hline & 0.60 & 0.63 & 0.71 & 0.47 \\
\hline APE & 0.31 & 1.71 & 1.19 & 2.51 \\
\hline SPE & 0.52 & 3.83 & 1.30 & 4.16 \\
\hline
\end{tabular}

\section{1-Propanol + Toluene + Dibutylether}

From the Table 2, it is evident that the ultrasonic velocities calculated by Nomoto's relation deviate from 0.09 to $0.47 \%$, IMR shows a deviation of 0.29 to $3.73 \%$, IDR deviate by 0.09 
to $1.69 \%$ and finally CFT makes a deviation of 0.2 to 1.18 over the entire concentration of 1-propanol. It is observed from the values of APE \& SPE (Table 2), the results for 1-propanol system in the order of NR, CFT, IDR and IMR.

\section{1-Butanol + Toluene +Dibutylether}

Table 2 shows, the deviation for the ultrasonic velocities calculated by NR, IMR, IDR and CFT in the range of 0.05 to $0.72 \% 0.06$ to $0.62 \%, 0.71$ to $1.88 \%$ and 0.28 to $1.39 \%$ respectively. From Table 2, it is evident that the theories agree well with the experimental values in the order of IMR, NR, CFT and IDR.

\section{1-Pentanol + Toluene + Dibutylether}

The value of percentage deviation (Table 2) for system III using NR is in the range of 0.01 to $1.24 \%$, IMR in the range of 0.10 to $10.1 \%$, then the IDR is of 0.20 to $1.69 \%$ and finally CFT is of 0.47 to $10.6 \%$. From the above results it is observed that the theories in the order of NR, IDR, IMR and CFT.

\section{Conclusion}

Ultrasonic velocities predicted using NR, IMR, IDR and CFT were compared with experimentally measured velocity values at $303 \mathrm{~K}$ in the three component mixtures of dibutylether and toluene with $n$-alkanols. Among the four theories, it is found that the NR is in best suited with the experimental velocity values.

\section{References}

1. Ulagendran U, Kumar R, Jayakumar S and Kannappan V, J Molecular Liquids, 2009, 148, 67.

2. Vasantharani P, Muthu Shailaja S, Kannappan A N and Ezhil Pavai R, J Appl Sci., 2008, 8(12), 2329-2332.

3. Pandey J D, Pantr N, Agarwal N and Shika, Acustica, 1989, 68, 225.

4. Rama Rao G V, Viswantha Sarma A, Siva Rama Krishna J and Rambabu C, Indian J Pure Appl Phys., 2005, 43, 345-354.

5. Agnihotri P K and Adgaonkar C S, Ultrasionics, 1989, 27, 248.

6. Kannappan A N, Arumugam V and Vasantharani P, J Curr Sci., 2003, 3(2), 277-280.

7. $\quad$ Nomoto O J, J Phys Soc., Japan, 1958, 13, 1528.

8. Van Dael W and Vargeel E, Proceedings of the 1st International Conference on Calorimetry and thermodynamics, Warsaw, 1969, 555.

9. Kalidoss M. and Srinivasamoorthy R, J Pure Appl Ultrason., 1997, 19, 9.

10. Sumathi. T and Uma Maheswari, Indian J Pure Appl Phys., 2009, 47, 782. 


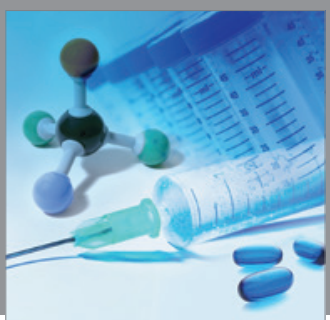

International Journal of

Medicinal Chemistry

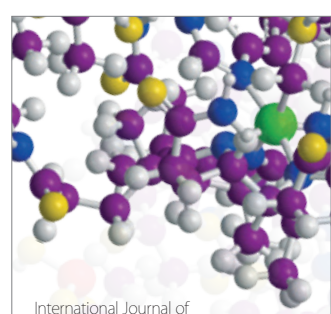

Carbohydrate Chemistry

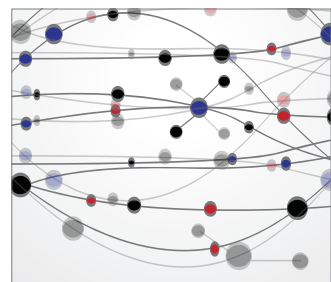

The Scientific World Journal
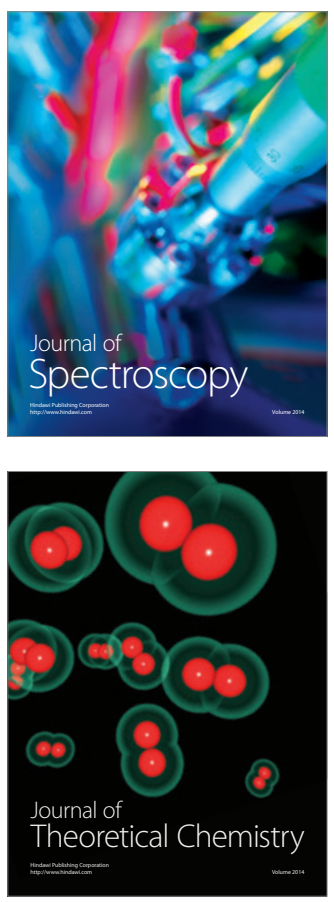
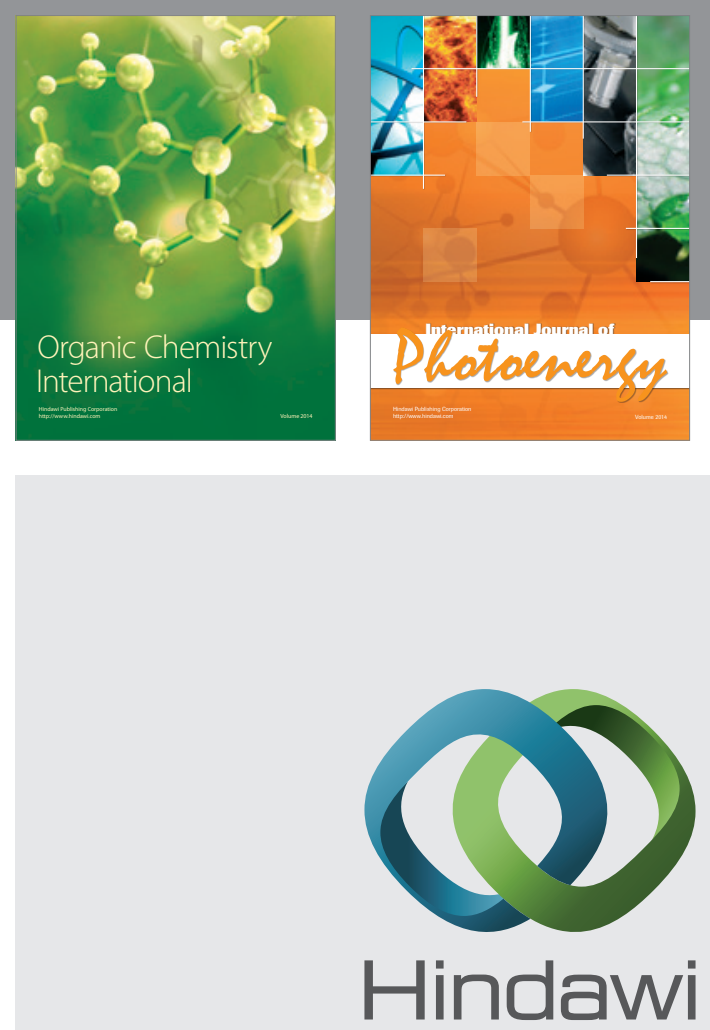

Submit your manuscripts at

http://www.hindawi.com
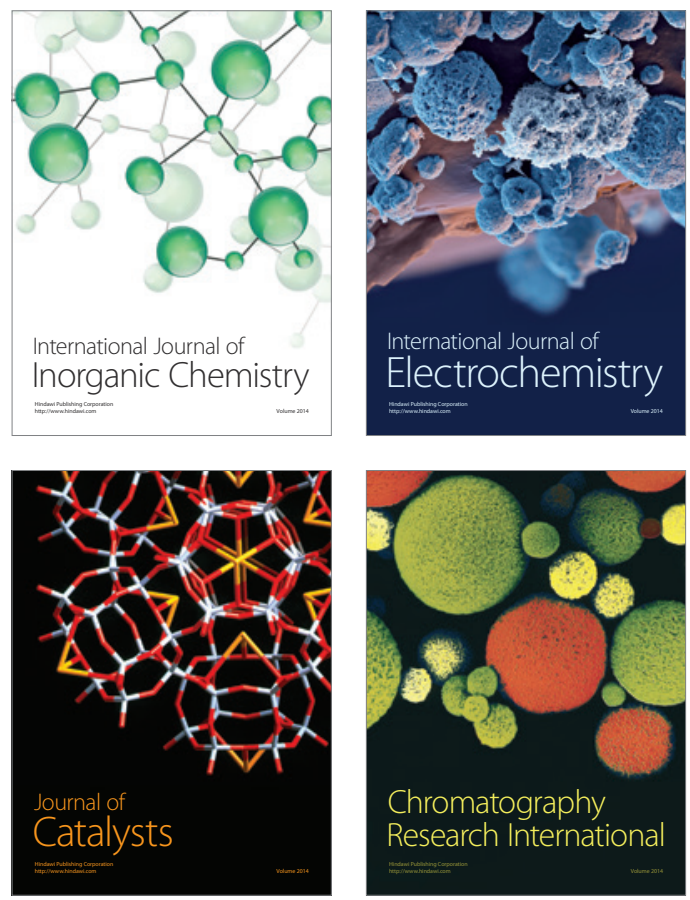
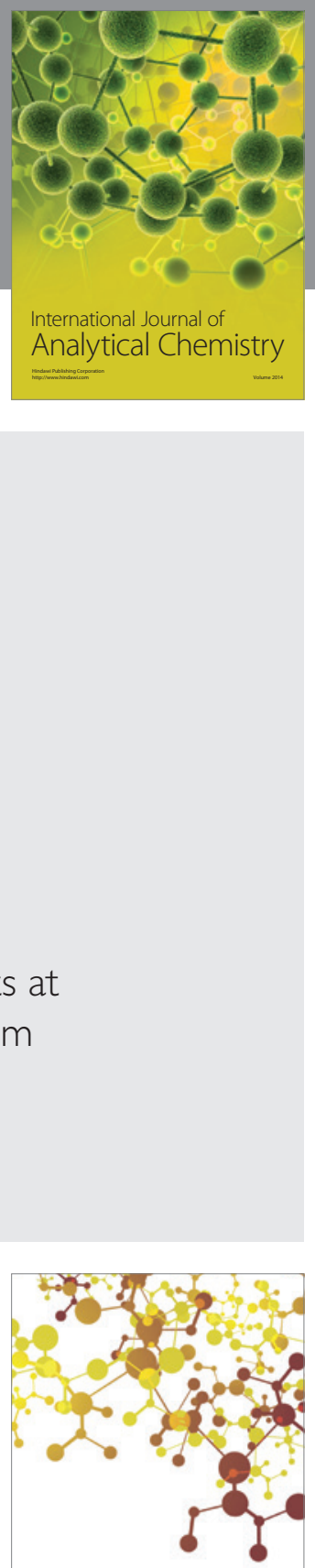

Journal of

Applied Chemistry
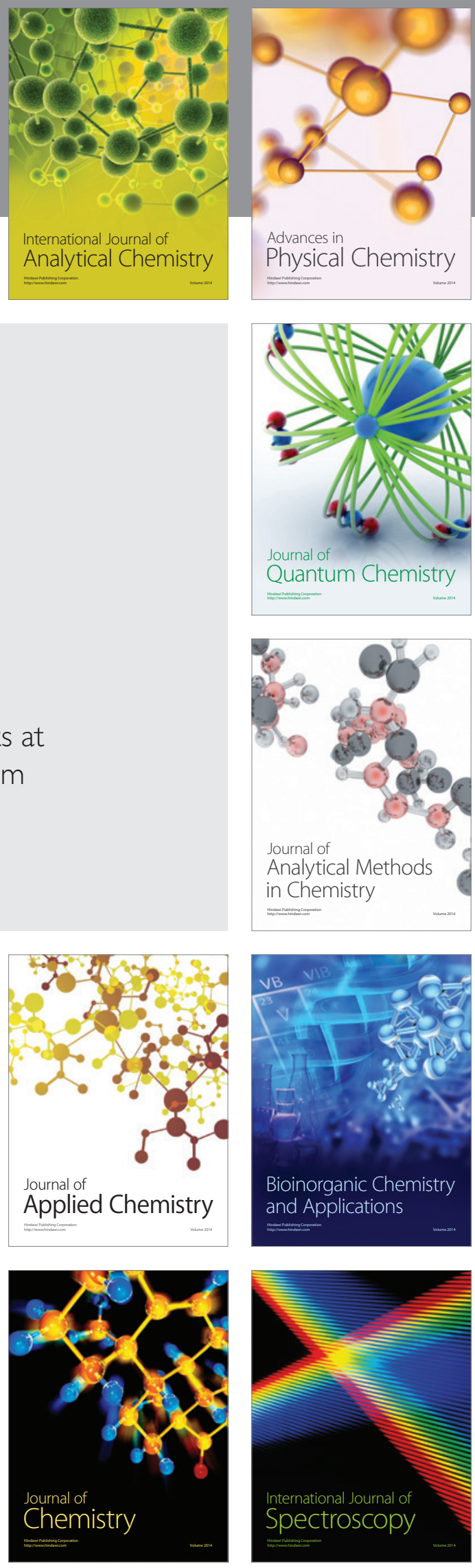